\title{
THE EROSION PROPERTIES OF POLYANHYDRIDES
}

\author{
LISA SHIEH $^{1}$, ACHIM GÖPFERICH $^{1,2}$ AND ROBERT LANGER $^{1}$ \\ 1. Rm E25-342, Department of Chemical Engineering, Massachusetts \\ Institute of Technology, 77 Massachusetts Ave., Cambridge, MA 02139 \\ 2. Current address: Universität Erlangen-Nümberg, Lehrstuhl für Pharmazeutische \\ Technologie, Cauerstrasse 4, 91054 Erlangen, Germany
}

\begin{abstract}
Bioerodible polymers play an important role in parenteral drug delivery. They combine the advantage of controlling drug release and being absorbed by the body after fulfilling their task. The mechanism which controls drug release is the erosion of polymer bulk. In order to understand and control the release it is, therefore, essential to know the erosion mechanism of a biodegradable polymer. We found that the polyanhydrides obey a distinct erosion pattern. An erosion zone (the region where material loss occurs) moves from the surface to the center of the discs. The monomers of which these copolymers are composed also seem to play an important role in degradation and erosion.
\end{abstract}

\section{INTRODUCTION}

Polyanhydrides are a class of bioerodible polymers that were developed specifically for controlled release drug applications. They are one of few synthetic degradable systems with regulatory approval for use in human clinical trials. However, to be better able to control drug release from a biodegradable polymer, we must first understand how the polymer erodes. In this paper, we describe studies investigating the erosion mechanism of the newly developed Poly (Fatty Acid Dimer: Sebacic Acid) \{p(FAD:SA)\} polyanhydride, which has been approved by the Food and Drug Administration for human clinical trials (see Figure 1).

Figure 1: Chemical structure of Poly (Fatty Acid Dimer: Sebacic Acid)

\section{P(FAD-SA)}

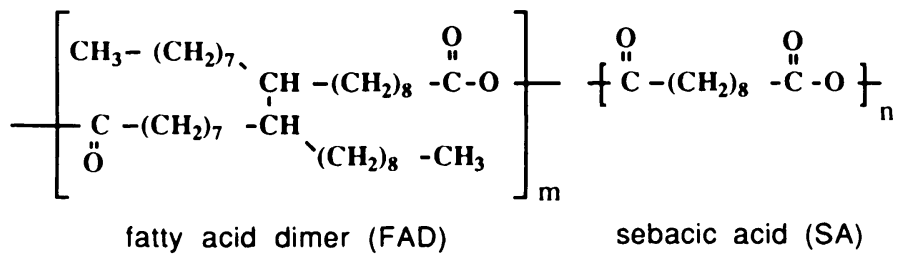


We began by visualizing the $\mathrm{p}(\mathrm{FAD}: \mathrm{SA})$ erosion process using light microscopy. The overall SA erosion of different monomer composition p(FAD:SA) copolymers was then examined to determine what effect copolymer properties had on polymer erosion. Differences in erosion patterns were explained by investigating factors which affect the erosion process. These include water penetration into the polymer matrix, degradation by anhydride bond hydrolysis, and dissolution and diffusion of interior degradation products (such as SA oligomers or monomers) to the polymer matrix surface. Monitoring SA release gives an idea of how a drug may release from the polymer as well. Since a surface eroding device is often desirable for controlled release systems (polymers undergoing surface erosion can provide easily controllable and constant drug release rates, and protect the drug from the harsh in-vivo environment), we also conducted studies to elucidate the type of erosion (surface vs bulk) p(FAD:SA) was undergoing.

\section{MATERIALS}

P(FAD:SA) was synthesized by melt polycondensation [1]. P(FAD:SA) copolymer discs (of $14 \mathrm{~mm}$ diameter, $2.7 \pm 0.1 \mathrm{~mm}$ thickness) were received as a gift from Nova Pharmaceuticals (Baltimore, MD). The polymers were of weight composition p(FAD:SA) 20:80, 50:50, 70:30 and $\mathrm{MW}=40,000$.

\section{METHODS}

\section{Light microscopy:}

Cross sections were cut with a razor blade from p(FAD:SA) 20:80 and p(FAD:SA) 50:50 discs at different erosion stages and examined under a light microscope (Wild Makroskop M420) at 25x.

\section{Erosion study:}

Erosion studies were conducted in $20 \mathrm{ml}$ of $0.1 \mathrm{M} \mathrm{pH} 7.4$ phosphate buffer at $37^{\circ} \mathrm{C}$ with constant shaking at 120 RPM. Effect of copolymer composition was studied using discs of $p(F A D: S A)$ 20:80, p(FAD:SA) 50:50, and p(FAD:SA) 70:30. Effect of device thickness on erosion rate was studied using p(FAD:SA) 50:50 discs of 0.68, 0.98, 1.40, and $1.67 \pm 0.01 \mathrm{~mm}$ thickness (measured using a micrometer). The buffer was changed frequently enough (at least once a day) to approximate perfect sink conditions. The buffer solutions were analyzed by reversed phase ion-pair high pressure liquid chromatography (Hewlett Packard 1090). The column used was a poly(styrenedivinylbenzene) reversed phase HPLC column (Hamilton, PRP-1), and the mobile phase consisted of acetonitrile in aqueous $0.05 \mathrm{~mol} / \mathrm{L}$ tetrabutylammonium phosphate (Waters, Pic A) at $\lambda=210 \mathrm{~nm}$.

\section{Hydrolysis of anhydride bond:}

The hydrolysis of three different copolymer compositions (p(FAD:SA) 20:80, p(FAD:SA) 50:50, p(FAD:SA) 70:30) was examined. At various time points, polymer samples were removed from release media, dissolved in chloroform and film cast onto $\mathrm{NaCl}$ plates. The outer "erosion zone" of the polymer was scraped from the inner intact zone and analyzed separately. The IR analysis was done with a Nicolet Magna-IR Spectrometer 550. The anhydride bond has a characteristic doublet occuring at 1800- 
1850 and $1740-1790 \mathrm{~cm}^{-1}$. Degradation was monitored by the carboxylic acid peak at $1700-1725 \mathrm{~cm}^{-1}$.

\section{Crystallinity:}

Thermal analysis of polymer samples of p(FAD:SA) 20:80, p(FAD:SA) 50:50: and p(FAD:SA) 70:30 was determined with a Perkin-Elmer DSC-7 Differential Scanning Colorimeter at a heating rate of $10^{\circ} \mathrm{C} / \mathrm{min}$. Copolymer crystallinity was calculated from the heat of fusion using the relation from [2].

\section{Water uptake:}

Samples of p(FAD:SA) 20:80, p(FAD:SDA) 50:50, and p(FAD:SA) 70:30 were placed in $\mathrm{pH} 7.4$ phosphate buffer at $37^{\circ} \mathrm{C}$; one was removed every 24 hours. Each was then dropped into liquid nitrogen and stored frozen until time of analysis, where they were dissolved in chloroform and titrated for water content using a Mettler DL18 Karl Fischer titrator with Hydranal solvent (Riedel-deHaen).

\section{RESULTS AND DISCUSSION}

Initial studies involved visualizing the $\mathrm{p}(\mathrm{FAD}: \mathrm{SA})$ erosion process. Light microscopy indicates an outer whitish "erosion zone" in the p(FAD:SA) 20:80 which grows from the outside towards the inside of the polymer disc. As the erosion zone progresses, disc thickness decreases. This is in contrast to $p(C P P: S A)$ where previous studies [3] reported no thickness change during erosion. The erosion zone in p(FAD:SA) 50:50 appears as a clear, adhesive, viscous substance on the discs's surface which also grows toward the polymer interior. As the FAD monomer ratio of the p(FAD:SA) copolymer is increased, the erosion zone's appearance and viscosity tend toward the properties of the FAD monomer. The erosion zone is thought to be formed when SA monomers (or oligomers) dissolve and diffuse out of the polymer matrix. The zone presumably includes insoluble degradation products, such as FAD monomer. Even after complete SA depletion from the device, there are still FAD remnants present. The presence of the erosion zone plays an important role in erosion and drug release because any water or monomer must diffuse through this eroded layer.

Evidence of certain surface eroding characteristics are present when the effect of device thickness on erosion was examined [4] (see Figure 2). The erosion rate of polymer matrices is independent of disc thickness until the erosion zones reach the disc center (and then thicker devices erode over a longer time). In addition, Karl Fischer water uptake results indicate very little water in the polymer bulk during polymer degradation. During the erosion process, the most hydrophilic copolymer, p(FAD:SA) $20: 80$, never exceeded $5 \mathrm{wt} \%$ water in the bulk. The more hydrophobic polymers p(FAD:SA) 50:50 and p(FAD:SA) 70:30 never exceeded $3 \mathrm{wt} \%$ and $1 \mathrm{wt} \%$ water in the interior during the erosion period. This indicates that there was very little free water in the polymer bulk. What little water that may have been present must have reacted almost instantaneously with the anhydride bond. This is in contrast with a purely bulk eroding system, where the hydrolysis reaction is often slower than water uptake, resulting in large percentages of water (sometimes up to $60 \mathrm{wt} \%$ [5]) in the polymer bulk. 
Figure 2: Effect of disc volume on SA erosion rate

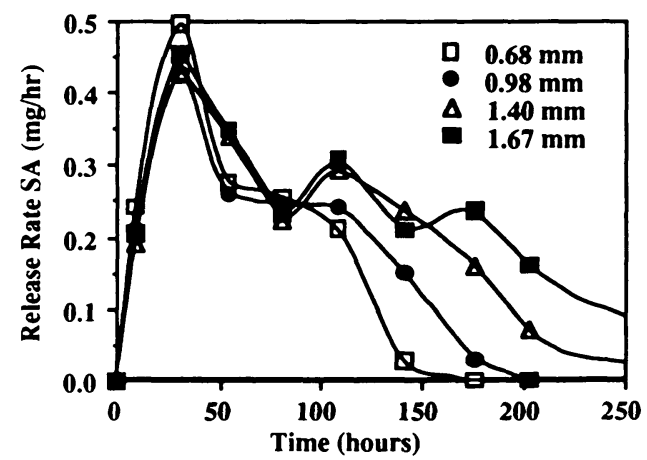

The p(FAD:SA) 50:50 eroded over the longest period (see Figure 3). It was thought that increasing the more hydrophobic monomer (FAD) of the copolymer may result in slower erosion due to further inhibition of water penetration. However, further increases up to $70 \mathrm{wt} \%$ actually decreased the erosion period. This is in contrast from what has been reported with the p(CPP:SA) polyanhydride. Leong et al. [6] have reported that they could obtain a wide range of CPP erosion rates (1 week to several years) by increasing the monomer ratio of CPP to SA. However, Goepferich et al. [7] have found that increasing CPP monomer content (although extending CPP release) does not actually affect $S A$ release from $p(C P P: S A)$. SA release from both $p(C P P: S A)$ 20:80 and $\mathrm{p}(\mathrm{CPP}: \mathrm{SA})$ 50:50 was about equal (over a time period of 7 days for $1 \mathrm{~mm}$ thick discs).

Figure 3: Effect of monomer composition on SA erosion

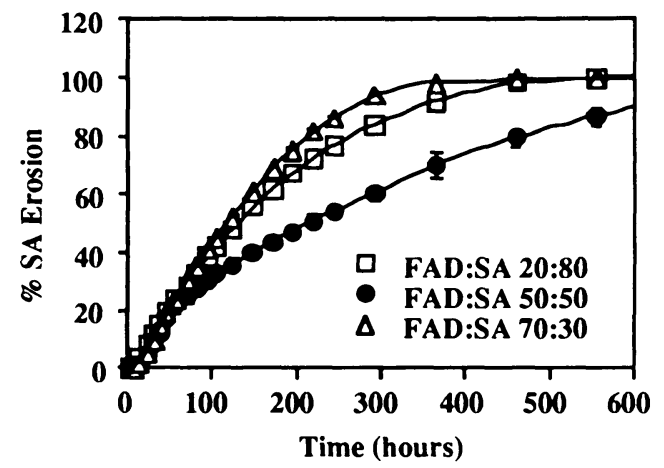

A possible explanation for the phenomenon seen in $\mathrm{p}(\mathrm{FAD}: \mathrm{SA})$ erosion is due to polymer hydrophobicity and crystallinity. As the copolymer FAD component increases, the polymer not only becomes more hydrophobic but also more amorphous. Differential Scanning Colorimetry (DSC) studies indicate that the polymer degree of crystallinity decreases with increasing FAD monomer content [4] (see Figure 4). Hydrophobicity inhibits water penetration into the polymer matrix, but amorphous domains are more vulnerable to hydrolytic attack [6,7], and therefore degrade more easily than crystalline regions. These two opposing copolymer properties may compromise the range of 
degradation rates that can be achieved by varying the monomer ratio in the p(FAD:SA) copolymer.

Figure 4: Crystallinity as function of monomer composition

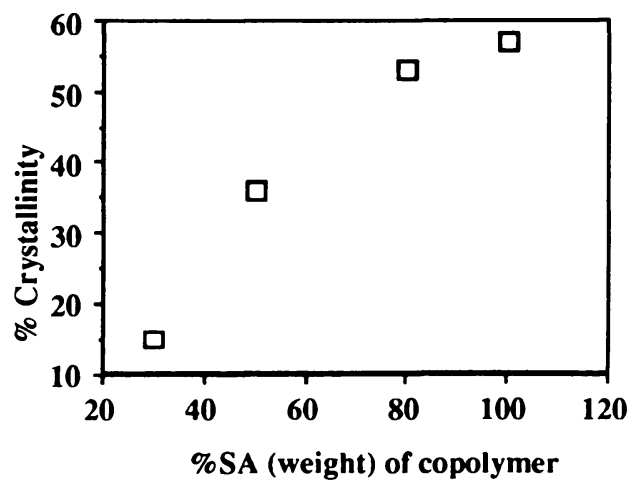

Correlating anhydride bond hydrolysis with appearance of SA in solution proved how important diffusion of the monomer/oligomer through the erosion zone is. Although the anhydride bonds of p(FAD:SA) 70:30 have completely hydrolyzed in 5 days, only $55 \%$ of the SA had appeared in solution. P(FAD:SA) 50:50 is completely hydrolyzed in 11 days, and only $55 \%$ of the SA had appeared in solution. The FAD content of the outer zone may be a diffusional barrier to the interior product diffusing out. In contrast for higher SA content copolymers, p(FAD:SA) 20:80 is completely degraded in 13 days and almost all of the SA (90\%) has appeared in solution. The more porous p(FAD:SA) 20:80 erosion zone may provide less of a barrier to the SA diffusing out compared to p(FAD:SA) 50:50 and p(FAD:SA) 70:30 erosion zones of higher (more insoluble) FAD content. The structure/composition of this erosion zone (which is related to copolymer monomer composition) does play an important role in overall erosion of the polymer device.

\section{CONCLUSIONS}

It appears that choice of monomers plays a role in the copolymer physical properties and erosion characteristics. It affects copolymer crystallinity, anhydride bond hydrolysis, and monomer dissolution and diffusion out of the polymer matrix. These processes all contribute to the overall polymer erosion pattern. We have also demonstrated that $\mathrm{p}$ (FAD:SA) has some surface eroding characteristics, such as the presence of an erosion zone, independence of erosion rate on device thickness, and low water content in the polymer interior during erosion.

\section{ACKNOWLEDGEMENTS}

The authors thank Dr. Janet Tamada for valuable discussions, Irene Chen and Judy Pang for technical assistance, and Dr. Avi Domb and Dr. Manoj Maniar for providing 
polymers. The financial support of NIH Grant\# CA5257 and a NSF Graduate Fellowship is also acknowledged.

\section{REFERENCES}

[1] Domb, A., and Maniar, M., Absorbable Biopolymers Derived from Dimer Fatty Acids, J. Polym Sci, 31, 1275-1285, 1993.

[2] Mathiowitz, E., Amato, C., Dor, Ph., and Langer, R., Polyanhydride Microspheres: 3. Morphology and Characterization of Systems Made by Solvent Removal, Polymer, 31, 547-555, 1990.

[3] Tamada, J., and Langer, R., Erosion Kinetics of Hydrolytically Degradable Polymers, Proc. Natl. Acad. Sci., 90, 552-556, 1993.

[4] Shieh, L., Tamada, J., Chen, I., Pang, J., Domb, A., and Langer, R., Erosion from a New Family of Biodegradable Polyanhydrides, submitted.

[5] Shah, S. S., Cha, Y., and Pitt, C.G., Poly(glycolic acid-co-DL-lactic acid): diffusion or degradation controlled drug delivery, J. Controlled Release, 18, 261-270, 1992.

[6] Leong, K. W., Brott, B.C., and Langer, R., Bioerodible polyanhydrides as drugcarrier matrices. I. Characterization, degradation, and release characteristics, J. Biomed. Mater. Res., 19, 941-955, 1985.

[7] Gopferich, A., and Langer, R., The Influence of Microstructure and Monomer Properties on the Erosion Mechanism of a Class of Polyanhydrides, J. Polym. Sci., 31, 2445-2458, 1993. 